\title{
APLICACIÓN DEL EXCEL PARA LA FORMULACIÓN DE RACIONES EN GANADO VACUNO LECHERO E IMPORTANCIA DE INDICADORES AL MOMENTO DE FORMULAR UNA RACIÓN
}

\section{APPLICATION OF EXCEL FOR RATION IN DAIRY CATTLE AND IMPORTANCE OF INDICATORS WHEN FORMULATING A RATION}

\section{Cesar Alexander López Ramos'; Victor Israel Tello Alarcón'; Victor Cesar Ramos Pacheco'; Frank Wagmer Manrique Flores'}

\section{RESUMEN}

Objetivo: Dar a conocer las aplicaciones y herramientas que tiene el Excel, que ordenadas en función de la formulación de Raciones y sumada con indicadores adecuados al análisis de alimentación ganadera, nos dará resultados más precisos, proporcionando a los ganaderos, técnicos y profesionales interesados, una herramienta informática de fácil uso, libre acceso y de bajo costo. Materiales y Métodos: Se utilizó el programa EXCEL como matriz del programa propuesto Easy Feed debido a que este se encuentra en toda computadora hasta la actualidad en conjunto con el complemento SOLVER, que fue usado para la programación lineal que da como resultado la formulación al mínimo costo y otras fórmulas basadas en literatura Internacional dedicada a la nutrición de ganado bovino lechero. Resultados: Las comparaciones efectuadas con el programa Mixit2, dieron resultados similares en relación a la formulación de raciones al mínimo costo, siendo el programa propuesto, el que permite utilizar otros indicadores para la afinación de la ración final. Conclusiones: Ambos programas pueden trabajar al mínimo costo o con programación lineal, la diferencia del programa propuesto es que este tiene otros indicadores que han sido desarrollados con el avance de la nutrición lechera a largo del tiempo por instituciones dedicadas a esta labor, estos indicadores no pueden ser insertados dentro del mixit2, por ser un programa de fuente cerrada, y los cuales en la actualidad son recursos a tomar en cuenta al momento de realizar un balance de raciones, cabe recordar que ambos programas dan como resultado lo que el operario le condicione en las restricciones, pero para mejorar estas condiciones tanto de nutrientes como de ingredientes, se proponen otras variables a considerar en el programa propuesto.

Palabras claves: Formulación, Raciones, Ganado lechero, Excel, Solver, Programas, Easy Feed.

\section{ABSTRACT}

Objective: This work was developed with the aim of publicizing the applications and tools you have the Excel, which ranked according to the formulation of rations and combined with appropriate indicators to the analysis of livestock feed, we will give more accurate results, providing farmers, technicians and professionals concerned, a software tool easy to use, free access and inexpensive. Materials and Methods: EXCEL program was used as the matrix of the proposed program Easy Feed because this is in any computer to date in conjunction with the Solver add-in, which was used for linear programming resulting formulation to the minimum cost and other formulas based on international literature on nutrition of dairy cattle. Results: The comparisons made with Mixit2 program, gave similar results in relation to the formulation of rations at minimum cost, with the proposed program, which lets you use other indicators to the pitch of the final ration. Conclusions: Both programs can work at minimal cost or linear programming, unlike the proposed program is that it has other indicators that have been developed with the progress of milk nutrition over time by institutions dedicated to this task, these indicators do not They can be inserted into the mixit2, as a program of closed source, and which today are resources to consider when making a balance rations, remember that both 
programs result in requiring the operator conditional him the restrictions, but to improve these conditions both nutrients and ingredients, other variables are proposed to be considered in the proposed program.

Keywords: Formulation, rations, Dairy Cattle, Excel,Solver, Programs, Easy Feed.

\section{INTRODUCCIÓN}

Actualmente la producción lechera mundial es una actividad altamente competitiva, ya que el productor lechero debe ser más eficiente, produciendo leche de mejor calidad y menor costo, que es lo que exige el mercado. "Se prevé que en el 2050 la demanda de carne y leche mundial aumente en un $73 \%$ y $58 \%$ respectivamente, en relación con los niveles del 2010 (...)" (FAO, 2011), y para Perú esto no sería la excepción por ello la industria lechera nacional debe buscar mejorar diversas debilidades, las cuales afectan la rentabilidad de sus establos.

Uno de los grandes problemas en la industria lechera nacional de medianos productores es la deficiente nutrición y los altos costos de los insumos dentro de la alimentación, siendo esta, la alimentación la que conlleva el $60 \%$ a $70 \%$ de los gastos de producción, a esto se le suma la falta de una herramienta informática que facilite la elaboración de una ración económica y de alta calidad nutricional. Los pequeños y medianos ganaderos productores de leche son los más afectados ya que no cuentan con una herramienta informática que les facilite el trabajo. En el mercado local existen herramientas informáticas de formulación de raciones, pero su uso está muy limitado a establos grandes debido a su elevado costo de compra.

\section{Objetivo:}

Dar a conocer las aplicaciones y herramientas que tiene el Excel, que ordenadas en función de la formulación de Raciones y sumada con indicadores adecuados al análisis de alimentación ganadera, nos dará resultados más precisos, proporcionando a los ganaderos, técnicos y profesionales interesados, una herramienta informática de fácil uso, libre acceso y de bajo costo.

\section{DEFINICIÓN DE PROGRAMA.-}

Un programa informático es un conjunto de instrucciones que una vez ejecutadas realizarán una o varias tareas en una computadora. Sin programas, estas máquinas no pueden funcionar. Al conjunto general de programas, se le denomina software, que más genéricamente se refiere al equipamiento lógico o soporte lógico de una computadora digital. Otra definición es que es un conjunto de instrucciones $u$ órdenes que indican a la máquina las operaciones que ésta debe realizar con unos datos determinados. En general, todo programa indica a la computadora cómo obtener unos datos de salida, a partir de unos datos de entrada. (Wikipedia, 2013).

Existen diferentes programas dentro del mercado informático, como RationMix.2012, ZMix, Zlac, ASODOBLE, ZOOTECH2012, Feedsoft, Dairy Live, entre otros, pero se describirán el programa más usado en nuestro medio, MIXIT2, el cual estaría a mayor alcance para los ganaderos conocedores de este, y el que más se asemeja al programa creado es uno hecho en Excel 2000 en Colombia denominado "FORMUNAL".

\section{1.-FORMUNAL}

Este programa fue desarrollado aplicando herramienta SOLVER de la hoja de cálculo Microsoft EXCEL 2000. Este procedimiento permite introducir un número ilimitado de restricciones que pueden ser aplicadas tanto a los nutrientes como a las materias primas seleccionadas de acuerdo a las necesidades del usuario.

El programa fue diseñado de tal manera que es posible mezclar simultáneamente hasta 13 materias primas para satisfacer las restricciones establecidas para un máximo de siete nutrientes diferentes, mostrando permanentemente el valor nutricional de los alimentos seleccionados, el resultado de la formulación en porcentaje (\%), y el costo de la misma. El programa posee tres ventanas, en una de las cuales es posible formular raciones para no rumiantes (aves de postura, aves de engorde y cerdos); en la segunda, se puede formular raciones para ganado de leche, en tanto que en la tercera, se maneja la base de datos del programa. Para el caso particular de la formulación de ración es para ganado lechero, se introdujeron las ecuaciones propuestas por el informe de National Research Council de 2001 sobre ganado lechero para estimar el valor proteico y energético de los alimentos. El uso de este programa permite la formulación de raciones a mínimo costo de una manera ágil y muy didáctica. (ASODOBLE, 2012).

\section{2.- MIXIT2}

MIXIT-2 consiste en catorce programas de computadora que almacenan información de los ingredientes usados en la alimentación animal y calcula mezclas balanceadas al mínimo costo para diferentes especies de animales. La mezcla de alimentación al mínimo costo es calculado por programación-lineal aplicando también sencillas bases de datos. Las características principales de MIXIT-2 son: 
Hasta 57 nombres de nutrientes con sus unidades de medida tal como (\%), (IU/G), (Mcal/kg), la cual puede ser seleccionada y posteriormente ser cambiada. Se pueden ingresar cientos de ingredientes para ser utilizado, dependiendo de la capacidad de disco. Nuevos ingredientes pueden ser añadidos o cambiados posteriormente.

Cualquier grupo de nutrientes (llamado 'Lista de nutrientes' o Nlist) puede ser seleccionada y restringida.

La composición y contenido nutricional de cualquier combinación de ingredientes puede ser impreso. El disco de datos acepta la información como "alimento fresco" o "alimento en base seca". Los pesos en hornada y pesos de báscula pueden ser impresos.

Calcula utilizando (o enfrentando) una 'Lista de nutrientes' a una 'Lista de ingredientes', se obtendrá una ración al mínimo costo.

Los siguientes puntos pueden ser mostrados en pantalla o ser impresos: todos los nombres de ingredientes y sus precios respectivos, todos los datos de los ingredientes, la composición de una mezcla y todas las listas de ingredientes y nutrientes.

Después de encontrar una ración al mínimo costo se puede redondear a una cantidad de Kg determinada e imprimir el contenido nutritivo de la mezcla. Los pesos de hornada y de báscula pueden ser impresos.

Los precios de oportunidad de los ingredientes alimenticios y los costos de los nutrientes de cualquier mezcla de mínimo costo pueden ser mostrados en la pantalla o ser impresos.

Se puede usar el sistema inglés o el sistema métrico (ACADEMIA, 2015).

\section{Easy Feed:}

Es un programa elaborado en Hoja de cálculo EXCEL inicialmente en EXCEL 2010 y actualizado en EXCEL 2013, este utiliza distintas funciones de Excel, la herramienta Solver, y Macros Básicas que en su conjunto automatizan a EASY FEED. El programa formula en kilos por animal al día, lo cual los otros programas no realizan debido a que trabajan en porcentaje, y siendo esto una limitante debido a que no te permite realizar otros cálculos que afinan la ración.

El programa determina el consumo de materia seca por una fórmula matemática. Esta es una combinación de varios autores (...), (NRC. 2001), en la cual usa tres variables principales que son la producción promedio, el peso promedio, y los días en leche, que reemplazando nos da el consumo de materia seca en kilos por animal al día, siendo este la principal variable para poder formular.

El programa propuesto formula y muestra la ración directamente en kilos por animal, su costo se muestra en soles por día, soles por kilogramo de materia seca y materia fresca, muestra en tiempo real al momento de formular otras variables a tomar en cuenta como FDN del forraje, Consumo condicionado por el FDN, relación forraje concentrado, relación PDR/CNF, Digestibilidad de la Materia Seca. Los límites de ingredientes están en kilos por animal, los límites de los requerimientos nutricionales están en las mismas unidades que cualquier otro programa (\%. Mcal $/ \mathrm{kg}$, $\mathrm{mg} / \mathrm{kg}, 1000 \mathrm{Ul} / \mathrm{KG}$ ), o que las entidades de investigación nutricional de esta especie.

Los indicadores que se utilizan son fórmulas desarrolladas por instituciones dedicadas a esta labor, siendo las siguientes: Digestibilidad de la materia seca (NRAES. 1995), Fibra detergente neutra del forraje (NRC. 2001), Consumo de materia seca condicionado por la fibra detergente neutra, digestibilidad de la Materia Seca (S, Calsamiglia. 1997), Relación carbohidratos no fibrosos con proteína degradable en el rumen (Sandra L Rueda. 2006)

\section{CONTRASTE ENTRE PROGRAMAS}

Se utilizó el software Mixit2 versus Easy Feed (programa propuesto), para realizar un fórmula para vacas en producción de 30 litros de leche, peso promedio de 600 kg y 200 días en leche.

Los requerimientos nutricionales Propuestos para ambos programas serían:

•Energía (Mcal/kg): 1.68-1.7

-Ca (\%): $0.58-0.8$

-Proteina (\%): 16.7 - 17

$\bullet P(\%): 0.37-0.6$

-Fibra (\%): 17

•E.E. (\%): $2.5-5$

-FDN (\%): 38 max

-C.N.F (\%): $30 \mathrm{~min}$

-FDA (\%): $18 \mathrm{~min}$

Los ingredientes a utilizar para ambos programas serian:

-Úrea

-Melaza

- Torta de soya

-Carbonato de calcio

-Afrecho

-Bicarbonato

-DDGS

-Pasta algodón

-Soya integral

-Suplamin Difos

-Sal

- Grasa protegida

-Maíz molido
- Chala chocleada 
Tabla 1. En esta tabla se muestra la cantidad de ingredientes en porcentaje.

L E A S T - C O S T F E E D M I X INGREDIENTS

16) CHALA CON CHOCLO

7) afrecho

6) maiz molido

1) Pasta algodon

5) torta soya

14) DDGS

12) carbonato $\mathrm{Ca}$

4) melaza

10) bicarbonato na

2) soya integral

21) SUPLAMIN DIFOS

13) sal

3) urea

11) grasa protegida

$0.38 / \mathrm{KG}$ (WET)

0. 95/KG (DRY)

39. $60 \%$ DRY MATTER
LARGEST COST 0.37

\section{AS FED}

$78,614 \%$

$6.675 \%$

$4.675 \%$

$4.400 \%$

$2.843 \%$

$0.900 \%$

$0.810 \%$

$0.528 \%$

$0.200 \%$

$0.151 \%$

$0.084 \%$

$0.060 \%$

$0.048 \%$

$0.011 \%$

$100.000 \%$

\section{PER TON}

$786.14 \%$

$66.75 \%$

$46.75 \%$

$44.00 \%$

$28.43 \%$

$9.00 \%$

$8.10 \%$

$5.28 \%$

$2.00 \%$

$1.51 \%$

$0.84 \%$

$0.60 \%$

$0.48 \%$

$0.11 \%$

100.00

Tabla 2. En esta tabla se muestra las restricciones de ingredientes en porcentaje.

I NGREDIENT RESTRICTIONS

\begin{tabular}{lrrrr}
\hline $\begin{array}{l}\text { INGREDIENTS } \\
\text { (INGREDIENTES) }\end{array}$ & $\begin{array}{c}\text { MINIMUN } \\
(\%)\end{array}$ & $\begin{array}{r}\text { ACTUAL } \\
(\%)\end{array}$ & $\begin{array}{r}\text { MAXIMUM } \\
(\%)\end{array}$ & COST \\
\hline 1) Pasta algodon & --- & 10.000 & 10.000 & $2.91951 \mathrm{E}-03$ \\
2) Soya integral & --- & 0.344 & 2.000 & \\
3) urea & --- & 0.120 & 0.120 & $3.786374 \mathrm{E}-02$ \\
4) melaza & --- & 1.000 & 1.000 & $7.715649 \mathrm{E}-03$ \\
5) torta soya & --- & 6.533 & -- & \\
6) maiz solido & --- & 10.506 & --- & $5.345166 \mathrm{E}-03$ \\
7) afrecho & --- & 15.000 & 15.000 & \\
11) grasa protegida & 0.500 & 0.025 & 0.100 & $1.677729 \mathrm{E}-02$ \\
10) bicarbonato na & --- & 0.500 & --- & $1.100049 \mathrm{E}-02$ \\
13) sal & --- & 0.150 & 0.150 & \\
12) carbonato Ca & --- & 2.000 & 2.000 & $4.796753 \mathrm{E}-04$ \\
14) DDGS & --- & 51.610 & -- & \\
16) CHALA CON CHOCLO & 0.207 & --- & \\
21) SUPLAMIN DIFOS & 0.090 & & & \\
\hline
\end{tabular}


Tabla 3. En esta tabla se muestra las restricciones de Nutrientes

NUTRIENT RESTRICTIONS

\begin{tabular}{lccccc}
\hline \multicolumn{1}{l}{ NUTRIENTS } & MINIMUN & ACTUAL & MAXIMUM & COST \\
\hline 5) PROT & $\%$ & 16.700 & 16.700 & 17.000 & $1.388695 \mathrm{E}-02$ \\
6) FC & $\%$ & 15.000 & 20.116 &.-- & \\
7) FDN & $\%$ & --- & 38.000 & 38.000 & .0173203 \\
8) FDA & $\%$ & 18.000 & 21.777 & --- & .0302235 \\
9) Ca & $\%$ & 0.580 & 0.800 & 0.800 & .3698087 \\
10) P & $\%$ & 0.370 & 0.370 & 0.600 & .3698087 \\
11) EN. lact & Mca/kg & 1.600 & 1.600 & 1.700 & $9.496929 \mathrm{E}-03$ \\
12) $\mathrm{EE}$ & $\%$ & 2.500 & 2.500 & 5.000 & $5.317794 \mathrm{E}-03$ \\
13) $\mathrm{CNF}$ & $\%$ & 30.000 & 33.389 & $-\cdots$ & \\
\hline
\end{tabular}

Tabla 4. En esta tabla se muestra la cantidad de ingredientes en base al requerimiento de materia seca.

\begin{tabular}{lcc}
\hline \multicolumn{1}{c}{$\mathbf{F}$ E E D M I X } & P R I C E & A M O U N T \\
\hline CHALA CON CHOCLO & 0.16 & 45.6551 \\
afrecho & 0.73 & 3.8764 \\
maiz molido & 1.21 & 2.751 \\
Pasta algodon & 1.28 & 2.5556 \\
torta soya & 2.08 & 1.6513 \\
DDGS & 1.22 & 0.5227 \\
carbonato Ca & 0.20 & 0.4704 \\
melaza & 0.50 & 0.3067 \\
bicarbonato na & 3.00 & 0.1162 \\
soya integral & 1.80 & 0.0879 \\
SUPLAMIN DIFOS & 6.25 & 0.0485 \\
sal & 0.25 & 0.0348 \\
urea grasa protegida & 1.44 & 0.0279 \\
\multicolumn{1}{c}{ TOTAL AMOUNT } & 5.67 & 0.0061 \\
\multicolumn{1}{c}{ TOTAL COST } & &.--1. \\
\hline
\end{tabular}


Tabla 5. Imagen donde se muestra las restricciones de ingredientes.

Fórmula con Easy Feed: Para determinar el consumo de materia seca se usa la fórmula del NRC 2001 y nos da un consumo de 23 kilos de materia seca por animal por día.

\begin{tabular}{|c|c|c|c|c|c|c|}
\hline$\%$ M.S. & Minimos & Maximos & M. Fresco & M. Seca & INGREDIENTES & PRECIO \\
\hline 10.47 & & 3.00 & 2.71 & 2.41 & AFRECHO & 0.73 \\
\hline 10.29 & & & 2.60 & 2.37 & TORTA DE SOYA & 2.08 \\
\hline 13.54 & & 3.50 & 3.50 & 3.12 & MAÍZ MOLIDO & 1.21 \\
\hline 7.83 & & 2.00 & 2.00 & 1.80 & SOYA INTEGRAL & 1.8 \\
\hline 0.82 & & & 0.19 & 0.19 & CARBONATO DE CALCIO & 0.2 \\
\hline 0.41 & & & 0.10 & 0.09 & SAL & 0.25 \\
\hline 0.00 & & & 0.00 & 0.00 & BICARBONATO & 3 \\
\hline 0.17 & 0.04 & & 0.04 & 0.04 & SUPLAMIN DIPOS & 6.25 \\
\hline 0.35 & & & 0.11 & 0.08 & MELAZA & 0.5 \\
\hline 0.00 & & 0.00 & 0.00 & 0.00 & UREA & 1.44 \\
\hline 0.00 & & & 0.00 & 0.00 & PASTA ALGODÓN & 1.28 \\
\hline 0.29 & & 0.07 & 0.07 & 0.07 & GRASA PROTEGIDA & 5.67 \\
\hline 3.83 & & 1.00 & 1.00 & 0.88 & DDGS & 1.22 \\
\hline 0.00 & & & 0.00 & 0.00 & Vacio & \\
\hline 0.00 & & & 0.00 & 0.00 & Vacio & \\
\hline 0.00 & & & 0.00 & 0.00 & Vacio & \\
\hline 0.00 & & & 0.00 & 0.00 & Vacio & \\
\hline 0.00 & & & 0.00 & 0.00 & Vacio & \\
\hline 0.00 & & & 0.00 & 0.00 & Vacio & \\
\hline 0.00 & & & 0.00 & 0.00 & Vacio & \\
\hline 0.00 & & & 0.00 & 0.00 & Vacio & \\
\hline 0.00 & & & 0.00 & 0.00 & Vacio & \\
\hline 52.00 & & & 46.00 & 11.96 & Chala con Choclo & 0.16 \\
\hline 0.00 & & & 0.00 & 0.00 & Vacio & \\
\hline 0.00 & & & 0.00 & 0.00 & Vacio & \\
\hline 0.00 & & & 0.00 & 0.00 & Vacio & \\
\hline 0.00 & & & 0.00 & 0.00 & Vacio & \\
\hline 0.00 & & & 0.00 & 0.00 & Vacio & \\
\hline 100 & & & 58.3 & 23.0 & & \\
\hline
\end{tabular}

Tabla 6. Imagen donde se muestra las restricciones de ingredientes.

\begin{tabular}{|c|c|c|c|c|c|c|c|c|c|c|c|c|c|c|c|c|c|}
\hline & MS Prot. & EN(lact) FC & FDN & $\mathrm{Ca}$ & CNF & E.E. & Potasi & o Sodic & o Cloro & FDA & Prot By Pas & s VACIO & $P$ & PDR & Azufre & Cobalto & Vit.D \\
\hline MAXIMO & $23 \quad 17$ & 1.7 & & 0.8 & & 5 & & & & & & & 0.6 & & & & \\
\hline MINIMO & 23.016 .7 & 1.6817 & 36 & 0.58 & 30 & 2.5 & & & & 18 & & & 0.37 & 63 & & & \\
\hline OBTENIDO & 23.016 .96 & 1.6819 .78 & 36.38 & 0.58 & 38.06 & 4.55 & $\begin{array}{ll}5 & 1.40\end{array}$ & 0.18 & 0.44 & 20.71 & 35.31 & 0.00 & 0.45 & 63.00 & 0.18 & 0.06 & 1107.83 \\
\hline
\end{tabular}

Tabla 7. Imagen donde se muestra los cuadros de relación forraje-concentrado y la relación carbohidratos no fibrosos - proteína degradable en el rumen.

\begin{tabular}{lr}
\hline \multicolumn{2}{c}{ RELACION FORRAJE - CONCENTRADO } \\
\hline Forraje & 52.00 \\
Concentrado & 48.00 \\
\hline
\end{tabular}

\begin{tabular}{lc}
\hline CNF & 38.06 \\
\hline Relación CNF - PDR & 3.56 \\
\hline$\%$ de PDR de la Prot. Total & 10.68 \\
\hline
\end{tabular}


Tabla 8. Imagen donde se muestra los cuadros de FDN del forraje, consumo condicionado por el FDN y Digestibilidad de la Materia Seca.

\begin{tabular}{lc}
\hline FDN del Forraje & 27.04 \\
\hline Consumo x FDN & 19.79 \\
\hline DIGEST. De la Materia Seca & 72.77 \\
\hline
\end{tabular}

\section{Contraste entre ambos programas}

Ambos programas pueden trabajar al mínimo costo o programación lineal, y como se puede notar ambos tienen resultados similares variando mínimamente en el costo debido a que uno formula en porcentaje y el otro en kilos por animal al día, lo que hace la diferencia es que el programa "EASY FEED" tiene otros indicadores que han sido desarrollados con el avance de la nutrición lechera a largo del tiempo por instituciones dedicadas a esta labor, estos indicadores en la actualidad son recursos a tomar en cuenta al momento de realizar un balance de raciones.

Tanto el mixit2 como el programa propuesto "EASY FEED" son Herramientas las cuales son una ayuda al momento de formular, pero esto no indica que las fórmulas obtenidas sean las mejores, ya que existen diferentes factores al momento de formular una ración. Cabe recordar que ambos programas dan como resultado lo que el operario le condicione en las restricciones, pero para mejorar estas condiciones tanto de nutrientes como de ingredientes, se proponen otras variables a considerar como FDN del forraje, consumo condicionado por el FDN, relación Forraje Concentrado, relación Proteína Degradable en el Rumen y Carbohidratos No Fibrosos, entre otras funciones más que se pueden implementar.

\section{DISCUSIÓN}

RATIONMIX 2012, ZMIX, ZLAC, ASODOBLE, ZOOTECH2012, FEEDSOFT y otros, hablan de programas de alta eficiencia en cuanto a formulación de raciones se refiere, son programas que formulan al mínimo costo en porcentaje, evalúan formulas realizadas por otros programas, algunos permiten cambiar la base de datos de sus ingredientes, siendo por estas características y otras mas no descritas los que los hacen,programas de excelencia, la desventaja es que son muy costosos, siendo los grandes ganaderos los únicos en poder acceder a ellos. Otras particularidades es que son de fuente cerrada, lo cual no permite implementar otras funciones, tiene fecha de caducidad, lo cual llegada la fecha de expiración, el programa no funciona o te exige una compra de una nueva versión. El programa propuesto tiene todas las funciones beneficiosas, siendo la principal la de poder implementarse cualquier otra función o formula nutricional que se desee agregar, que ayude a mejorar la elaboración de las raciones. No existe ningún costo para su adquisición, ya que se encuentra libre en internet, no tiene fecha de expiración debido a que Excel solo se actualiza según las versiones que vallan lanzando Microsoft Windows.

\section{CONCLUSIONES}

La finalidad de este artículo no es generar problemas si no alternativas para los ganaderos que no pueden acceder a una herramienta informática para la formulación de raciones y el desarrollar un programa informático utilizando una hoja de cálculo EXCEL para formular raciones para el ganado vacuno lechero, es una herramienta válida, porque elabora una ración óptima y al mínimo costo ya que se contrasto con un programa de alto nivel informático, y se logró una herramienta de fácil uso, de libre acceso y de bajo o nulo costo, cabe recordar que es mejor crear herramientas propias, debido a que se puede implementar mejoras conforme el avance de la Nutrición y Alimentación Animal.

\section{RECOMENDACIONES}

Se recomienda buscar implementar al programa otras mejoras que puedan servir de guía al nutricionista o ganadero, para poder elaborar fórmulas de mejor calidad.

Se recomienda buscar la viabilidad de este programa en otras especies, ya que los programas para otras especies son similares debido a que todos trabajan con programación lineal o formulación al mínimo costo, agregándole funciones que ayuden a mejorar la formulación de raciones de la especie para la que se formule.

\section{REFERENCIAS ELECTRÓNICAS}

FAO. (2011). World Livestock 2011 Livestock in food security. ROMA: FAO. Disponible en http://www.fao.org/publications/es/

The Northeast Regional Agricultural Engineering Service NRAES. (1995). Dairy Reference Manual. PENNSYLVANIA: CATHLEEN WALKER.

De la Alimentación a la leche: Comprendiendo la Función del Rumen. PEEN STATE. Disponible en http://extension.psu.edu/animals/dairy/nutrition

NUTRIENT REQUIREMENTS OF DAIRY CATTLE, 2001. National Research Council.

Calsamiglia, S. (1997). XIII Curso de Especialización avances en nutrición y alimentación animal, Nuevas bases para la utilización de la fibra en dietas de rumiantes. Disponible en www.fundacionfedna.org

(2006). Relación entre el flujo de proteína microbiana hacia el duodeno y algunos parámetros metabólicos y productivos en vacas lactantes de un hato lechero del Oriente Antioqueño. Disponible en:

http://www.scielo.org.co/scielo.php?pid=S0120$06902006000100004 \&$ script=sci_arttext

Feedsoft .2012; [1 página]. Disponible en http://www.feedsoft.com/es/productos/conceptos/

RationMix.2012; [2 páginas]. Disponible en http://www.rationmix.com/caracteristicas

Zootech.2012; [1 página]. Disponible en http://www.xootech.com.pe/productos.html

Asodoble.2012; [1 página]. Disponible en http://www.asodoble.com/servicios-en-linea.php.

Wiquipedia.2013; [2 páginas]. Disponible en http://es.wikipedia.or/wiki/Programa inform\%C3\% A1tico

Academia.2015; [39 páginas]. Disponible en https://www.academia.edu/3182631/Manual_de_B alanceo de Raciones al M\%C3\%ADnimo Costo _usando_Mixit-2 